\title{
A Hypergraph Approach to Conflict
}

M.B.M. de Koster

J.H.P. Paelinck

Erasmus University, Rotterdam

\section{Introduction}

In De Koster, Paelinck and Vossen (1983) a general form of choice models was presented in which a finite number of agents choose from a finite set of options or alternatives those that are considered by each agent to be "satisfying."

Generally we define a choice situation $\mathrm{C}$ as a pair $(\mathrm{B}, \Gamma)$, where $B=\left\{b_{1}, \ldots, b_{n}\right\}$ is the set of options, and $\Gamma=\left(E_{i} \mid i \varepsilon\{1,2, \ldots, m\}\right)$ is a family of subsets of $B$ (not necessarily distinct). $E_{i} \varepsilon \Gamma$ is called the choice set of agent $a_{i} \varepsilon A$, where $A=\left\{a_{1}, \ldots, a_{m}\right\}$ is the set of all agents.

We say there is a conflict between the agents if there is no option simultaneously satisfying all agents.

Note that, in a way, this model is related to "social choice" models (see e.g., Arrow, 1963; or more recently Moulin, 1983). However, we generally require less information about preferences of individual agents.

We will now state some possible conditions which the family of choice sets may, or may not, satisfy, and which are of special interest for the analysis. We divide them into three classes.

I. Conditions on individual choice sets

(i) $\quad \forall E \& \Gamma[|E| \geqslant 2]^{1}$

(ii) $\quad \forall E \in \Gamma[|E|=r]$ (so-called $r$-uniformity) 
II. Conditions on pairs of choice sets

(iii) $\forall \mathrm{E}_{\mathrm{i}}, \mathrm{E}_{\mathrm{j}} \in \Gamma\left[\mathrm{E}_{\mathrm{i}} \cap \mathrm{E}_{\mathrm{j}} \neq \emptyset\right]$ (pairwise agreement)

(iv) $\forall E_{i}, E_{j} \varepsilon \Gamma\left[i \neq j \rightarrow E_{i} \neq E_{j}\right]$

(v) $\forall E_{i}, E_{j} \varepsilon \Gamma\left[i \neq j \rightarrow E_{i} \not \subset E_{j}\right]$ (in that case $\Gamma$ is called a Sperner family, or antichain).

III. Conditions on the whole collection of choice sets

(vi) $\prod_{i=1}^{m} E_{i}=\emptyset$ (so there is a conflict)
(vii) $\exists S \subseteq B \quad \forall E \in \Gamma[E \nsubseteq S, E \cap S \neq \emptyset]$ (property $B)^{2}$

In De Koster, Paelinck and Vossen (1983) special interest was devoted to choice situations satisfying (i), (iii) and (iv). Such a choice model might describe, for example, the situation in which members of a government have to choose from a number of political alternatives $(n \geqslant 2)$. Because they belong to the same government, any two members will probably show some resemblance in their opinions, but because they have some degree of personality, no two opinions will be exactly the same; furthermore, all members will be flexible enough to have always more than one alternative in their choice sets (albeit similar ones!).

It is easy to see that, if $|B|=n$, and $\Gamma$ satisfies (i), (iii) and (iv), then $|\Gamma| \leqslant 2^{n-1}$ and that this upper bound can be attained. Now by theorem 3.1, following the structure of such a "maximal" family, which is known in part, has induced us to look into the "minimal" elements of $\Gamma$, that is, those elements that constitute a Sperner subfamily. ${ }^{3}$

Agents having choice sets constituting such a Sperner subfamily are very important, because each one is a representative of those agents who have a choice set containing his minimal set.

In this paper we study choice situations $C=(B, \Gamma)$, with $\Gamma$ satisfying (i), (iii) and (iv), maximum $|\Gamma|$, and other possible conditions. Many concepts are borrowed from a branch of mathematics called "hypergraph" theory (see Berge, 1970).

In section 2 we give a survey of some known extremal results ${ }^{4}$ for families of subsets of B satisfying some of the above mentioned restrictions and/or restriction (v).

In section 3 we characterize $C=(B, \Gamma)$, satisfyng (i), (iii), (iv) and $|\Gamma|=2|B|-1$, i.e., for maximal $\Gamma$, by means of the so-called base.

In section 4, we estimate the number of choice situations under these conditions and in section 5 we draw some conclusions. 


\section{Some Known Results in Hypergraph Theory}

In this section we will present some known results regarding the maximum size of families of subsets of an n-set $B$ which satisfy conditions mentioned in the introduction, especially the conditions (i), (iii), (iv) and (v); of course, an upper bound to the size of such a family exists only if the family satisfies (iv), that is, if the family is a set.

Definition 2.I Let $|\mathrm{B}|=\mathrm{n}, \mathrm{r} \varepsilon\{1,2, \ldots, \mathrm{n}\}$. By $\mathrm{K}_{\mathrm{h}}^{\mathrm{n}}$ we denote the set $\{S \subseteq B|\quad| S \mid=r\} ;$ by $\Lambda=\left\{A_{1}, \ldots, A_{m}\right\}$ we now denote a set of subsets of $B$.

One of the earliest results is due to E. Sperner (1928). n Theorem 2.1. Let $\Lambda$ satisfy (v). Then $m \leqslant\left[\frac{n}{2}\right]$.' Equality occurs only

$$
\text { if } \Lambda=\left\{\begin{array}{l}
\frac{k_{n}^{\frac{n}{2}}}{n+1} \\
\frac{n}{k_{n}^{2}} \text { or } \cdot k_{n}^{\frac{n-1}{2}}, \text { if } n \text { odd }
\end{array}\right.
$$

Now let us combine some conditions. The following theorem has been deduced from a theorem of Milner (1968), who proved a more general result.

Theorem 2.2. Let $\Lambda$ satisfy (iii) and (v). Then $m \leqslant\left(\left[\frac{n+2}{2}\right]\right)$.

Equality occurs only if $\Lambda=K_{n}\left[\frac{n+2}{2}\right]$ or, for $n$ even, if there exists $b \in B$ such that either $b \varepsilon A_{i}$ and $\left|A_{i}\right|=\frac{n}{2}$ or $b \notin A_{i}$ and $\left|A_{i}\right|$ $=\frac{n+2}{2}$, for all $A_{i} \varepsilon \Lambda$.

Note that the bound for $\mathrm{n}$ odd is the same as the bound in theorem 2.1. Theorem 2.3 (Erdös, Ko and Rado [1961]). Let $r \leqslant \frac{\mathfrak{n}}{2}$, and let $\Lambda$ satisfy (iii), (v), and let $\forall A \in \Lambda[|A| \leqslant r]$. Then $m \leqslant\left(\begin{array}{c}n-1 \\ r-1\end{array}\right)$. Equality occurs only if $\Lambda$ is the family of all sets having cardinality $r$ and containing a fixed $b \varepsilon B$.

In the latter case this means in our terminology that there is no conflict. A more recent result, which generalizes the preceding one is as follows:

Theorem 2.4 (Hilton [1974]). Let $1 \leqslant q \leqslant n-1$ and $1 \leqslant p \leqslant \min (q, n-q)$; let $\Lambda$ satisfy (iii), and let $\forall A \varepsilon \Lambda[p \leqslant|A| \leqslant q]$. Then

$$
\begin{aligned}
& \text { m }<\sum_{\substack{q \\
\text { m }}}\left(\begin{array}{c}
n-1 \\
i-1
\end{array}\right) \cdot \quad \text { If, additionally, } \Lambda \text { satisfies (vi), then } \\
& \sum_{i=p}\left[\left(\begin{array}{c}
n-1 \\
1-1
\end{array}\right)-\left(\begin{array}{c}
n-q-1 \\
i-1
\end{array}\right)\right] \cdot \mid
\end{aligned}
$$


For more extremal theorems, see Katona (1974).

Some of the theorems introduced in this section will help us understand the "base" concept discussed in the next section.

\section{Bases of Maximal Families Satisfying (i), (iii) and (iv)}

Suppose $|B|=n$, and that $C=(B, \Gamma)$ is a choice situation in which $\Gamma$ satisfies (i), (iii) and (iv) such that $|\Gamma|=2^{n-1}$. In De Koster, Paelinck and Vossen (1983) such a family $\Gamma$ is shown to be a so-called family of majorities, that is, $\Gamma$ satisfies (iv) and the conditions (a), (b) and (c) of the following theorem (see, for example, Monjardet [1978]).

Theorem 3.1. Let $\mathrm{C}=(\mathrm{B}, \Gamma)$ be a choice situation such that $\Gamma$ satisfies (iv). Then it follows that the following two statements are equivalent:

(1) $\Gamma$ satisfies (iii) and $|\Gamma|=2|B|-1$;

(2) $\Gamma$ possesses the following properties:
(a) $[A \in \Gamma] \Leftrightarrow\left[\begin{array}{lll}\bar{A} & \in\end{array}\right]$
(b) $\left[A \in \Gamma, A_{2} \supseteq A_{1}\right] \Rightarrow\left[A_{2} \varepsilon \Gamma\right]$
(c) $[A \in \Gamma] \Leftrightarrow[A \cap E \neq \emptyset$, for all $E \in \Gamma]$.

Remark 3.1. It is easy to show that condition (c) and the conditions (a) and (b) are equivalent.

Starting from this theorem, it is natural to look into the minimal members of such a family. This leads to the following definition.

Definition 3.1. Let $|B|=n$. A set $\Lambda$ of subsets from $B$ is called a base if $\Lambda$ satisfies (i), (iii), (v) and if

$|\operatorname{sp}(\Lambda)|=2^{n-1}$, where $\operatorname{sp}(\Lambda)=\{E \subseteq B \mid \exists \Lambda \subset \Lambda[E \supseteq A]\}$.

In other words $\operatorname{sp}(\Lambda)$ is the set of all subsets of $B$, containing a certain element of $\Lambda$.

For a set $\Lambda$ of subsets of $B$ and for $E_{i} \varepsilon \Lambda$, we could interpret

$\left\{E_{\varepsilon} \Lambda \mid E \supseteq E_{i}\right\}$ as the "rank and file" or adherence of agent $a_{j}$.

Examples 3.1.

(a) Let $B=\left\{b_{1}, \ldots, b_{6}\right\}$ and let

$$
\Lambda=\left\{\left\{b_{3}, b_{4}\right\},\left\{b_{3}, b_{5}\right\},\left\{b_{1}, b_{4}, b_{5}\right\},\left\{b_{2}, b_{4}, b_{5}\right\},\left\{b_{3}, b_{4}, b_{5}\right\},\right.
$$

Then $\Lambda$ is a base, as can be checked.

(b) Let $B=\left\{b_{1}, \ldots, b_{n}\right\}, n \geqslant 3$. Then again $\Lambda=\left\{\left\{b_{1} b_{2}\right\},\left\{b_{1}, b_{3}\right\}\right.$, $\left.\left\{b_{2}, b_{3}\right\}\right\}$ is a base. 
Remark 3.2. For a given choice situation $\mathrm{C}=(\mathrm{B}, \Gamma)$, with $\Gamma$ satisfying (i), (iii), (iv) and with $|\Gamma|=2|B|-1$, the elements of $\Gamma$ additionally satisfying (v) can be considered the choice sets of agents who can be seen as "decisive": for every agent $a_{i}$ with choice set $E_{i} \varepsilon \Gamma$, there exists a representative agent $a_{j}$, decisive for $a_{i}$, with choice set $E_{j} \varepsilon \Gamma$, such that every option in $E_{j}$ is also satisfying agent $a_{i}$.

Now by definition 3.1 every base determines a unique maximal family of choice sets satisfying (i), (iii) and (iv). The reverse is also true, as is shown by the following proposition, which is easy to prove.

Proposition 3.1. Let $\Gamma$ be a maximal family of subsets of $B$, satisfying (i), (iii) and (iv). Then $\Gamma$ contains exactly one base.

To characterize bases we need some definitions.

Definitions 3.2. Let $\mathrm{C}=(\mathrm{B}, \Gamma)$ be an arbitrary choice situation. A set $\mathrm{S} \varsigma^{\prime} \mathrm{B}$ is said to be free (stable) if it contains no choice set of a cardinal greater than one. The maximum cardinal of a free set is denoted by $B(C)$ and is called the stability number of $C$. The chromatic number, $\chi(C)$, is the smallest integer $k$ for which there exists a partition of $B$ into $k$ free sets.

$\beta(C)$ can be interpreted as the maximum cardinality that a group, or set of options, can have such that it does not contain a choice set, under condition (i). In other words, such that it does not contain all the elements satisfying a certain agent. $\chi(C)$ is the smallest number of groups into which the options can be divided such that not all options satisfying an agent are in a group. Note that $\chi(C)=2$ is the same as "property B" (condition [vii]), provided $\Gamma$ satisfies (i).

The following inequalities are known from hypergraph theory. Let $\mathrm{C}=(\mathrm{B}, \Gamma)$ bef a choice situation. Then

(a) $\chi^{(C) \beta(C)} \geqslant|B|$

(b) $\chi(C)+\beta(C) \leqslant|B|+1$

For a proof, see Berge (1970).

We have the following theorem.

Theorem 3.2. Let $\mathrm{C}=(\mathrm{B}, \Gamma$ be a choice situation where $\Gamma$ satisfies (i), (iii) and (v). Then $\Gamma$ is a base iff $\chi(C) \neq 2$.

Proof. Let $\Gamma$ be a base. Suppose $\chi(C)=2$. Then there exists $S \subseteq$ B such that for all $E \varepsilon \Gamma$, we have $E \notin S$ and $E \notin \bar{S} .^{6}$ Since $|\operatorname{sp}(\Gamma)|=2|\mathrm{~B}|-1$ we have, in view of Theorem 3.1, either $\mathrm{S} \varepsilon \operatorname{sp}(\Gamma)$ or $\overline{\mathrm{S}} \varepsilon \operatorname{sp}(\Gamma$, a contradiction. So $\chi(C) \neq 2$. For the reverse, let $|\operatorname{sp}(\Pi)<2| B \mid-1$. Then there exists $S \subseteq$ B such that $S \notin \operatorname{sp}(\Gamma)$ and $\bar{S} \notin \operatorname{sp}(\Gamma)$. Hence for all $E \varepsilon \Gamma$ we have $S \geq E$ and $\overline{\mathrm{S}} \supseteq \mathrm{E}$. So $\chi(\mathrm{C})=2$.

An immediate consequence of this theorem is that a base is maximal, in the sense that no more sets can be added without violating the restrictions of the theorem.

To prove the next theorem we need a lemma. 
Lemma 3.1. Let $\mathrm{C}=(\mathrm{B}, \Gamma$ be a choice situation, let $\Gamma$ satisfy (iii), and let $\chi(C) \geqslant 3$. Then there exist $E_{1}, E_{2} \varepsilon \Gamma$ such that $\left|E_{1} \cap E_{2}\right|=1$.

Proof. Let $\mathrm{A}_{1} \in \Gamma$ have the minimum cardinal. Now choose

$S \varepsilon \Gamma, b \varepsilon A_{1} \cap S$ and define $T:=A_{1} \backslash\{b\} . \chi(C) \neq 2$, so there exists $A_{2} \varepsilon \Gamma$ such that either $A_{2} \subseteq T$ or $A_{2} \subseteq \bar{T}$. However, $A_{2} \Phi T$, because $|T|<\left|A_{1}\right|=\min \{|A| \mid A \in \Gamma\}$. Hence $A_{2} \subseteq \bar{T}$

By (iii), $A_{2} \cap A_{1} \neq \emptyset$

Combination of (3.1) and (3.2) yields $A_{1} \cap A_{2}=\{b\}$.

Theorem 3.3. Let $\mathrm{C}=(\mathrm{B}, \Gamma$ be a choice situation where $\Gamma$ satisfies (iii). Then $\chi(C)=2$ or $\chi(C)=3$.

Proof. Suppose $\chi(C) \neq 2$. In view of lemma S.1 there exist $A_{1}, A_{2} \varepsilon \Gamma, b \in B$, such that $A_{1} \cap A_{2}=\{b\}$. Now define $S_{1}:=A_{1} \backslash\{b\}, S_{2}:=\{b\}, S_{3}:=B \backslash A_{1} \cdot\left\{S_{1}, S_{2}, S_{3}\right\}$ is a partition of $\mathrm{B}$ and, by (iii), for all

$\mathrm{E} \varepsilon \Gamma|\mathrm{E}|>1$ we have $\mathrm{E} \nsubseteq \mathrm{S}_{1}, \mathrm{E} \nsubseteq \mathrm{S}_{2}$ and $\mathrm{E} \nsubseteq \mathrm{S}_{3}$. So $\chi(C)=3$.

From the theorems 3.2 and 3.3 we now conclude

Corollary 3.1 Let $\mathrm{C}=(\mathrm{B}, \Gamma)$ be a choice situation and let $\Gamma$ satisfy (i), (iii), (v).

It follows that the following three statements are equivalent

(a) $\Gamma$ is a base

(b) $\chi(C) \neq 2$

(c) $\chi(C)=3$.

From this corollary it appears that for the choice sets, being a maximal family is equivalent to the fact that for every subdivision of the options into two groups there exists a "decisive agent" whose options belong completely to one group. We also know that there exists a subdivision into three groups such that this is not the case.

Of special interest to us are families $\Gamma$ that do not satisfy condition (vi), because they represent non-conflict choice situations. A family $\Gamma$ of subsets of an n-set $B$ is called a filter, with base $S \subseteq B$, if $\Gamma=\{E \subseteq B \mid E \supseteq S\}$. Clearly if $\Gamma$ is a filter and $C=(B, \Gamma)$, then $\chi(C)=2$. With the aid of the theorems 3.2 and 3.3 we can say something about the reverse.

Corollary 3.2. Let $\mathrm{C}=(\mathrm{B}, \Gamma$ be a choice situation where $\Gamma$ satisfies (iii), (iv) and $|\Gamma|=2^{n-1}(n=|B|)$. Then $\chi(C)=2$ iff $\Gamma$ is an ultrafilter (that is, $\Gamma=\{E \subseteq B \mid b \varepsilon E\}$, for some $b \varepsilon B$ ).

Proof. Let $\chi(C)=2$. Suppose $\Gamma$ additionally satisfies (i). Now let $\Lambda$ be the subfamily of $\Gamma$ satisfying (v). Then $\Lambda$ is a base, and given theorem 3.3, $\chi((B, \Lambda))=2$, which contradicts theorem 3.2. Thus there exists $\mathrm{E} \varepsilon \Gamma,|\mathrm{E}|<2$. By (iii), $\mathrm{E}=\{\mathrm{b}\}$, for some $\mathrm{b} \varepsilon \mathrm{B}$. Hence, by (iii) and $|\Gamma|=2^{\mathrm{n}-1}$, it follows that $\Gamma$ is an ultrafilter. 
Corollary 3.3. Let $C=(B, \Gamma$ be a choice situation where $\Gamma$ satisfies (i), (iii) and (iv). Then $\chi(C)=3$ iff $\Gamma$ contains a base.

In section 2, upper bounds were given to the number of sets in a base (theorems 2.2, 2.3 and 2.4). We now consider bases additionally satisfying (ii) $)_{r}$, so-called $r$-uniform bases. That is, every "decisive" agent has to choose exactly $r$ options.

Definition 3.3. $\mathrm{m}_{\mathrm{n}}(\mathrm{r})$ is the least integer for which there exist $\mathrm{m}_{\mathrm{n}}(\mathrm{r})$ sets $A_{i} \subseteq B,\left|A_{i}\right|=r$, with $1 \leqslant i \leqslant m_{n}(r)$, and $|B|=n$ which do not satisfy (vii) (property $B$ ).

Erdös (1969) proved:

Theorem 3.4. $m_{2 N-1}(r) \geqslant m_{2 N}(r) \geqslant 1 / 2\left(\begin{array}{c}2 N \\ N\end{array}\right) \cdot\left(\begin{array}{c}2 N-r \\ N-r\end{array}\right)^{-1}$

$$
\begin{aligned}
& m_{2 N+1}(r) \leqslant m_{2 N}(r) \leqslant N \quad\left(\begin{array}{c}
2 N \\
N
\end{array}\right) \cdot\left(\begin{array}{c}
2 N-r \\
N-r
\end{array}\right)^{-1} \\
& m_{2 n-1}(n)=m_{2 n}(n)=\left(\begin{array}{c}
2 n-1 \\
n
\end{array}\right)
\end{aligned}
$$

From the above theorem, theorem 2.5, and the simple fact that for a base condition (i) is equivalent with condition (vi). we have

Corollary 3.4. Let $|B|=n$, let $2 \leqslant r<\frac{n}{2}$ and let $\Lambda=\left\{A_{1}, \ldots, A_{m}\right\}$ be a base such that (ii) holds. Then

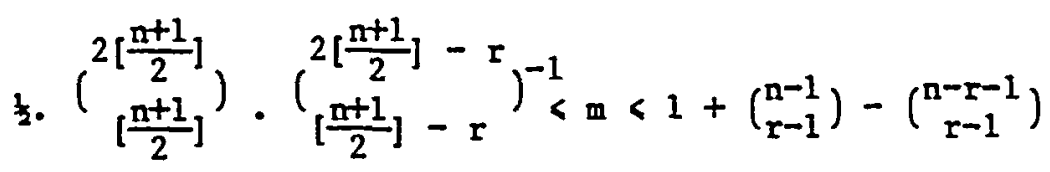

Note that, if (ii) $r$ does not hold, we generally can say only that $m \geqslant 3$. (For a base with $m=3$, see examples $3.1[b]$.) bases.

Finally, we give some examples and constructions of $r$-uniform Examples 3.2

(a) The Fano plane on seven points is a 3-uniform base (cf. Fig. 1). 


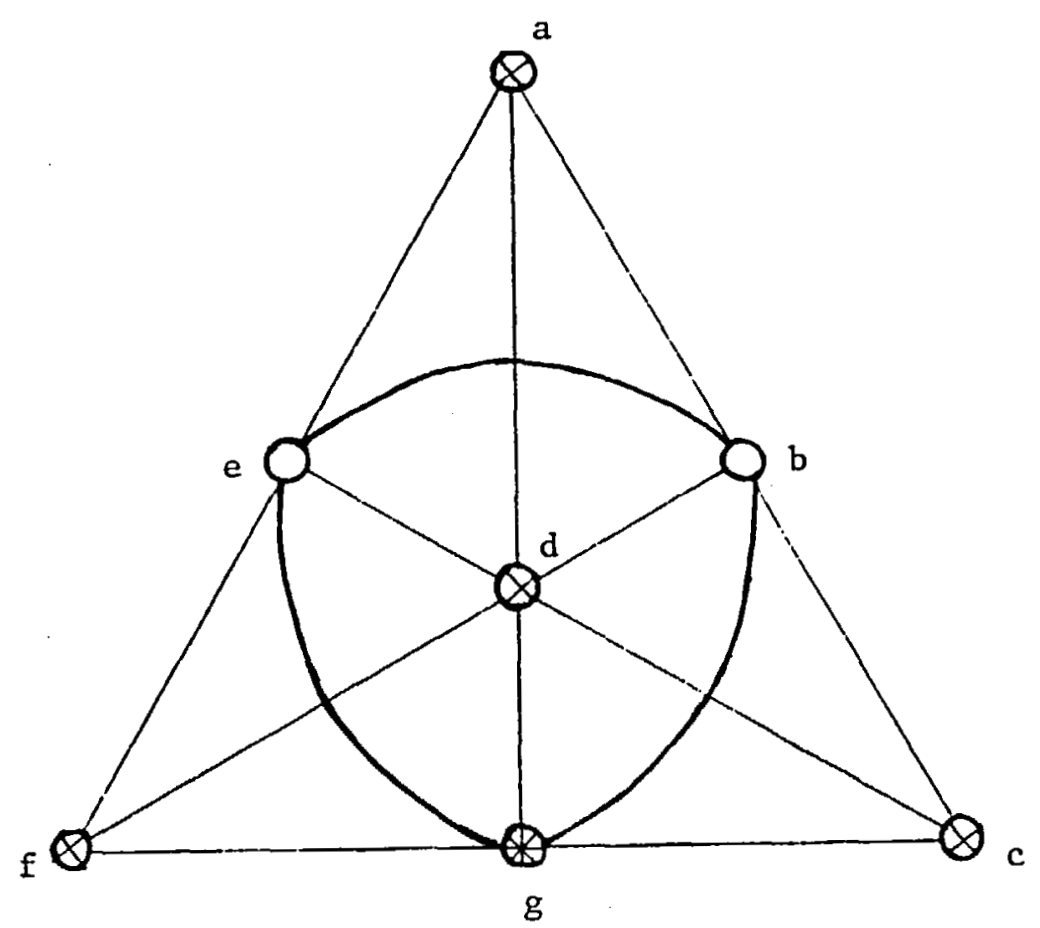

Figure 1: The Fano plane

The choice sets are: $\{a, b, c\},\{a, e, f\},\{a, d, g\},\{b, d, f\},\{b, e, g\},\{c, d, e\}$, $\{c, f, g\}$. The stability number is $\beta(C)=4$. In Figure 1 a 3 -coloration (partition) of the points is also given.

(b) $\mathrm{K}_{\mathrm{n}-1}$ is an $\mathrm{n}$-uniform base; in the proof one may use the following lemma.

Lemma 3.2. Let $|\mathrm{B}|=\mathrm{n}$, and let $\Lambda=K_{n}^{\mathrm{r}}$. Then $|\operatorname{sp}(\Lambda)|=\sum_{i=\mathrm{r}}^{n}\left(\begin{array}{l}\mathrm{n} \\ 1\end{array}\right)$.

Proof. Let $\mathrm{E} \subseteq \mathrm{B}$ be a set of cardinal $\mathrm{k} \geqslant \mathrm{r}$. Then $\Lambda$ contains a set $\mathrm{F}$, $|F|=r$, with $F \subseteq E$. Hence $E \varepsilon \operatorname{sp}(\Lambda)$.

(c) Let $|B|=n \geqslant 1 / 2\left(\begin{array}{c}2 r-2 \\ r-1\end{array}\right)+2 r-2$. Take a $2 r-2$-element subset $S$ of $B$. For each partition $P=\left\{S_{1}, S_{2}\right\}$ of $S$, with $\left|S_{1}\right|=\left|S_{2}\right|=r-1$, take a new option $x_{p} \varepsilon B \backslash S$. Now define $\Lambda$ to consist of all $r$-tuples from $S$ plus all r-tuples of the form $S_{1} \cup\left\{x_{p}\right\}$, where $P=\left\{S_{1}, S_{2}\right\}$ is a partition as constructed above. Then $\Lambda$ is an r-uniform base.

For more constructions, see Erdös and Lovász (1975). 


\section{The Number of Choice Situations Satisfying (i), (iii), (iv)}

In this last section we will consider only choice situations $C=(B, \Gamma)$ where $\Gamma$ satisfies (i), (iii), and (iv); and $|B|=n$. We will give an asymptotic formula for the logarithm of their number and an asymptotic estimate of the number of maximal families. We will estimate the number of non-conflicting families. Finally we will show that the fraction of nonconflicting families converges to 0 as $n$ tends to infinity. We will use the following notations:

$f(n):=$ the number of different families;

$g(n):=$ the number of different maximal families;

$h(n):=$ the number of different families not satisfying (vi).

By theorem 3.1 we know that every maximal family $\Gamma$ satisfying (i), (iii), (iv) and $|\Gamma|=2^{n-1}$ is uniquely characterized by its base. Now every base is a Sperner family, or antichain. Some results are available about the number of Sperner families. From them we can obtain an upper bound to the number of maximal families restricted as above, that is $g(n)$, the bound being

$$
2^{\left(\left[\begin{array}{l}
n \\
2
\end{array}\right]\right)\left(1+o\left(\log _{2} n / n\right)\right)}
$$

(see Kleitman and Markowsky [1975]). ${ }^{7}$

By a method due to Erdōs and Kleitman (1974) one can also obtain a lower bound for $\mathrm{g}(\mathrm{n})$.

Theorem 4.1. $\mathrm{g}(\mathrm{n}) \geqslant 2$

$$
\left(\left[\frac{n}{2}\right]\right)\left(\frac{1+o(1)}{2}\right)^{8}
$$

Proof. Let $\mathrm{b} \varepsilon \mathrm{B}$. First suppose $\mathrm{n}$ even. Divide all $\mathrm{n} / 2$-element subsets of

$B$ into two disjoint classes

$U:=\left\{E \subseteq B|| E \mid=\frac{n}{2}, b \in E\right\}, V:=\left\{E \subseteq B|| E \mid=\frac{n}{2}, b \in E\right\}$.

Now $V=\{E \subseteq B \mid \bar{E} \varepsilon U\}$. Mark $|U|=|V|=\left(\begin{array}{c}n-1 \\ \frac{p}{2}\end{array}\right)=\left(\begin{array}{l}n \\ \frac{n}{2}\end{array}\right) / 2$ So there exist $2^{\left(\frac{n}{2}\right) / 2}$ elements of $U$. Now each family $\Lambda$ determines a maximal family $\Gamma$, provided $n \geqslant 4$. Take $\Gamma:=$

$$
\begin{aligned}
& \left\{E \subseteq B|| E \mid>\frac{\mathfrak{n}}{2}\right\} \cup\left\{E \subseteq B|| E \mid=\frac{\mathfrak{n}}{2}, E \in \Lambda\right\} U \\
& \left\{E \subseteq B|| \bar{E} \mid=\frac{n}{2}, \quad \text { b } \varepsilon \bar{E}, \bar{E} \notin \Lambda\right\} .
\end{aligned}
$$


For $n$ odd we define $U:=\left\{E \subseteq B|| E \mid=\frac{n-1}{2}, \quad b \quad \varepsilon E\right\}$

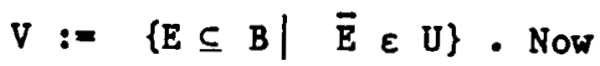

$$
|u|=|v|=\left(\frac{n-1}{2}\right)=\left(\frac{n-1}{n+1}\right) \sim \frac{1}{2}\left(\begin{array}{c}
n \\
{\left[\frac{n}{2}\right]}
\end{array}\right)
$$

There exist 2 different families $\Lambda$ made up of elements of $U$, and each family determines a maximal $\Gamma$ as follows:

$$
\begin{aligned}
& \Gamma:=\left\{E \subseteq B|E \in \Lambda,| E \mid=\frac{n-1}{2}\right\} \cup \\
& \quad\left\{E \subseteq B|| E \mid>\frac{n+1}{2}\right\} \cup\left\{E \subseteq B|\bar{E} \notin \Lambda,| \bar{E} \mid=\frac{n-1}{2}, b \varepsilon \bar{E}\right\} \\
& U\left\{E \subseteq B \mid \text { b } \varepsilon E,|E|=\frac{n+1}{2}\right\} .
\end{aligned}
$$

(To see that $|\Gamma|=2^{n-1}$, mark

that $\left|\left\{E \in \Gamma|| E\left|=\frac{n-1}{2} \quad \vee\right| E \mid=\frac{n+1}{2}\right\}\right|=\frac{(n-1)}{2}$.)

So in both cases we have: $g(n) \geqslant 2\left(\left[\frac{n}{2}\right]\right)^{\frac{1+o(1)}{2}}$

Now we can easily derive

Theorem 4.2. $f(n)=2^{2^{n-1}}(1+o(1))$.

Proof. Suppose $C=(B, \Gamma$ is a choice situation, given $\Gamma$ restricted as before and $|\Gamma|=2^{n-1}$. Now every subfamily of $\Gamma$ (except $\emptyset$ ) determines a choice situation restricted as above. On the other hand, all such families are subfamilies of maximal families. Hence, by the result of Kleitman and Markowsky (1975), it follows that

$$
2^{2^{n-1}} \cdot 2^{2^{n-1} \circ(1)}<f(n)<2^{2^{n-1}} \cdot 2^{\left(\left[\frac{n}{2}\right]\right)(1+o(1))}
$$


Therefore, since

$$
\left(\left[\frac{n}{2}\right]\right) \sim 2^{n} \cdot r \frac{2}{\pi n}=2^{n-1} \cdot \gamma \frac{8}{\pi n} \text {, we have }
$$

$f(n)=2^{2^{n-1}}(1+o(1))$.

Theorem 4.3. $\mathrm{h}(\mathrm{n})=0\left(\mathrm{n} .2^{2^{\mathrm{n}-1}}\right)$.

Proof. 1. Let $\Gamma$ be a family of subsets of B, satisfying (i), (iii), (iv), and not satisfying (vi). Then $\Gamma$ is contained in at least one ultrafilter. Since the number of different ultrafilters on $B$ is equal to $|B|=n$, we have $h(n) \leqslant n . s^{2^{n-1}}$.

2. Now suppose $\Lambda_{1}, \ldots, \Lambda_{\mathrm{n}}$ are the different ultrafilters on $\mathrm{B}$, with base $\left\{b_{1}\right\}, \ldots,\left\{b_{n}\right\}$ respectively. For all i $\varepsilon\{1,2, \ldots, n\}$, let

$\Gamma_{1}=\left\{E_{1} \mid j \in I_{1}\right\} \subset \Delta_{1} \backslash\left\{\left\{b_{1}\right\}\right\}$ be such that $\left|I_{1}\right|>2^{n-2}$. Then $\bigcap_{\varepsilon I_{1}}^{j} E_{I_{j}}=\left\{b_{1}\right\} \cdot$ (Suppose $\left.\mid \prod_{f \in I_{1}} E_{I_{j}}\right\}$. 2. Then for all

$f \in I_{1}$ we have: $\left\{x_{1}, y_{1}\right\} \leq E_{1 j}$, for some $x_{1}, \nabla_{1} \in B$. But then $\left|I_{1}\right|<2^{n-2}$, a contradiction.) This 1mplies for all

$1, j \in\{1,2, \ldots, n\}, 1 \neq j: \Gamma_{1} \neq \Gamma_{j}$. Hence $h(n)>n \cdot \sum_{1=2^{n-2}+1}^{2^{n-1}-1}\left(2_{1}^{n-1}-1\right)$

n. $\sum_{1=2^{n-2}+1}^{2^{n-1}-1}\left[\left(2_{1}^{n-1}\right)-\left(2_{1-1}^{n-1}-1\right)\right]$

$=n \cdot \sum_{1=2^{n-2}+1}^{2^{n-1}}\left(2_{1}^{n-1}\right)-n \cdot\left(2_{2^{n-1}}^{2^{n-1}}\right)-n \sum_{1=2^{n-2}}^{2^{n-1}-2}\left(_{1}^{2^{n-1}-1}\right)$

$=n \cdot\left[2^{2^{n-1}-1}-\left(2^{2^{n-1}}\right) / 2-1-2^{2^{n-1}-2}+1\right]$

$=\frac{n}{4} \cdot\left[2^{2^{n-1}}-2\left(\begin{array}{c}2^{n-1} \\ 2^{n-2}\end{array}\right)\right]$

$\sim \frac{\mathrm{n}}{4}\left[2^{2^{\mathrm{n}-1}}-\frac{2^{2^{\mathrm{n}-1}}}{\sqrt{\pi \cdot 2^{\mathrm{n}-4}}}\right] \sim \frac{\mathrm{n}}{4} \cdot 2^{2^{\mathrm{n}-1}}$ 
Our main goal in this section is to prove $\frac{h(n)}{f(n)} \rightarrow 0$ as $n$ tends to infinity. However, the estimate for $f(n)$, provided by theorem 4.1 , is insufficient for this purpose. Therefore we now prove

Proposition 4.1. $\mathrm{f}(\mathrm{a})>(1 / 3)$ n. $2^{2^{\mathrm{n}-1}}\left(\log _{3}(\mathrm{n}-1)-\frac{9}{2}\right)$.

Proof. Let $s \in\left[1,2, \ldots,\left[\frac{n-1}{2}\right]\right]$ and $n=\left[\frac{n}{2 s+1}\right](2 s+1)+a(n>3)$.

Let, for a $>0, P=\left\{s_{1}, \ldots, s_{\left[\frac{n}{28+1}\right]}, s\right\}$ be a partition of $B$, with $\left|s_{1}\right|=\ldots=\left|s_{\left[\frac{\mathfrak{n}}{2 s+1}\right]}\right|=2 s+1,|s|=a$. If $a=0$, then take $P=\left\{s_{1}, \ldots s_{\left[\frac{n}{2 s+1}\right]}\right\}$, with $\left|s_{1}\right|=\ldots=$ $\left|s_{\left[\frac{a}{2 s+I}\right]}\right|=2 s+1$. For all $1 \in\left\{1, \ldots,\left[\frac{n}{2 s+I}\right]\right\}$ let $1_{R_{2 s+1}^{s+1}} \subset \mathcal{T}\left(s_{1}\right)$ be the set of all s+1-tuples in $s_{1}$. Note $\left|\operatorname{sp}\left({ }^{1} x_{2 s+1}^{s+1}\right)\right|=2^{n-1}$.

We now construct different families of subsets of $B$, satisfying (i), (iii), and (iv) as follows:

For given $s \in\left\{1,2, \ldots,\left[\frac{n-1}{2}\right]\right\}, 1 \in\left\{1,2, \ldots,\left[\frac{n}{2 s+1}\right]\right\}$ a family consists of:

1. at least one element of $1_{\mathrm{k}^{s+1}}^{\mathrm{s}+1}$;

2. zero or more elements of $\operatorname{sp}\left({ }^{1} K_{2 s+1}^{s+1}\right) \backslash{ }^{1} k_{2 s+1}^{s+1}$

Now mark that the number of different families obtained in this way is equal to (s,i given):

$$
\begin{aligned}
& \left(2^{\left(\begin{array}{c}
2 s+1 \\
s+1
\end{array}\right)}-1\right) \cdot 2^{2^{n-1}}-\left(\begin{array}{c}
2 s+1 \\
s+1
\end{array}\right) \\
& =2^{2^{n-1}}-2^{2^{n-1}-\left(\begin{array}{c}
2 s+1 \\
s+1
\end{array}\right)}
\end{aligned}
$$

and that they all satisfy the restrictions. Mark also that for different $i$ and $s$ families obtained in this way are unequal. Hence,

$$
\begin{aligned}
& f(n)>\sum_{s=1}^{\left[\frac{n-1}{2}\right]}\left[\frac{n}{2 s+1}\right]\left(2^{2^{n-1}}-2^{2^{n-1}}-\left(\begin{array}{c}
2 s+1 \\
s+1
\end{array}\right)\right) \\
& =2^{2^{n-1}} \sum_{s=1}^{\left[\frac{n-1}{2}\right]}\left[\frac{n}{2 s+1}\right]-2^{2^{n-1}} \sum_{s=1}^{\left[\frac{n-1}{2}\right]}\left[\frac{n}{2 s+1}\right] 2^{-\left(\begin{array}{c}
2 s+1 \\
s+1
\end{array}\right)}
\end{aligned}
$$


Now $\left[\frac{n-1}{\sum_{s=1}}\right]\left[\frac{n}{2 s+1}\right]>\left[\frac{[-1}{2}\right]_{s=1} \frac{n-(2 s+1)}{2 s+1}=n\left[\frac{n-1}{2}\right] \frac{1}{2 s+1}-\left[\frac{n-1}{2}\right]$

Let $R_{m}:=1 / 3+1 / 5+1 / 7+\ldots+1 /(2 m+1)$ and let $p \in \mathbb{N}$ be such that $\frac{3^{p+1}-1}{2}>\left[\frac{n-1}{2}\right]>\frac{3^{p}-1}{2}$.

Then $\mathrm{R}_{\left[\frac{\mathrm{n}-1}{2}\right]}>\mathrm{R}_{\frac{3^{\mathrm{P}}-1}{2}}>1 \cdot \frac{1}{3}+3 \cdot \frac{1}{9}+\ldots+3^{\mathrm{P}-1} \cdot \frac{1}{3^{\mathrm{P}}}=\frac{\mathrm{P}}{3}$.

Hence $\sum_{s=1}^{\left[\frac{n-1}{2}\right]} \frac{1}{2 s+1}>\frac{p}{3}>\frac{1}{3}\left(\log _{3}\left(2\left[\frac{n-1}{2}\right]+1\right)-1\right)$.

Purthermore $\sum_{s=1}^{\left[\frac{n-1}{2}\right]}\left[\frac{n}{2 s+1}\right] 2^{-\left(\begin{array}{l}2 s+1 \\ s+1\end{array}\right)}<\frac{n}{3} \sum_{s=1}^{\left[\frac{n-1}{2}\right]} 2^{-\left(\begin{array}{c}2 s+1 \\ s+1\end{array}\right)}$

$$
<\frac{\mathrm{n}}{3} \sum_{s=0}^{\infty} 2^{-s}=\frac{2 \mathrm{n}}{3} \text {. }
$$

Substituting (4.2), (4.3) and (4.4) in (4.1) we obtain:

$$
\begin{aligned}
f(n) & >2^{2^{n-1}}\left(\frac{n}{3} \log _{3}\left(2\left[\frac{n-1}{2}\right]+1\right)-\frac{n}{3}-\left[\frac{n-1}{2}\right]-\frac{2 n}{3}\right) \\
& >2^{2^{n-1}}\left(\frac{n}{3} \log _{3}(n-1)-\frac{3}{2^{n}}\right) .
\end{aligned}
$$

Note that the lower bound to $\mathrm{f}(\mathrm{n})$ obtained in this proposition is far from the best possible. For instance applying the same idea also to the families constructed in the proof of theorem 4.1 increases the lower bound with a factor $f y$. The best bound we have obtained so far is:

$f(n)>n \cdot\left(\frac{3}{2}\right)^{n-1} \cdot 2^{2^{n-1}}(1+o(1))$.

However, the bound of proposition 4.1 is sufficient to prove our main result.

Corollary 4.1. $\lim _{\mathrm{n} \rightarrow \infty} \frac{\mathrm{h}(\mathrm{n})}{\mathrm{f}(\mathrm{n})}=0$

Proof. Theorem 4.3 and proposition 4.1 .

From this corollary we see that the fraction of non-conflicting families satisfying (i), (iii), and (iv) converges to 0 as $n$ tends to infinity. So, in our 
model, all families satisfying (i), (iii), and (iv) appear to be conflicting asymptotically.

This conforms to our intuition, which tells us that when there are many options, consensus is much more exceptional than conflict.

Note, however, that the construction used in the proof of proposition 4.1 has the drawback that in a certain family of choice sets the options may appear not chosen by any single agent. In practical reasoning, of course, such options would immediately be rejected.

\section{Conclusions}

For $0-1$ choice situations, with $n$ possible alternatives to choose from, where the agents agree pairwise, and all have different choice sets, we can draw the following conclusions.

If the number of agents is maximum under these restrictions, that is, $2^{\mathrm{n}-1}$, we can characterize the situation by theorem 3.1. Another possible description can be given by the chromatic number; this number is 2 or $3 ; 2$ in case of an ultra-filter (corollary 3.2) and 3 in case every agent chooses at least two options (corollary 3.3).

If the number of agents is smaller than $2^{\mathrm{n}-1}$, corollary 3.3 also states that the chromatic number is 3 if and only if the choice sets of the so-called "decisive agents" constitute a base, provided all agents choose at least two options.

In the last section we have estimated the number of $0-1$ choice situations and also the number of maximal non-conflicting choice situations. Thus we may conclude that the fraction of non-conflicting families converges to 0 as $n$ tends to infinity.

However, there remain some interesting questions. For instance, suppose one weakens the definition of non-conflicting situations and instead defines them as "the minimum cardinality of a subset of B, which has a non-empty intersection with every choice set is smaller than or equal to 2." Is it then true that the fraction of non-conflicting families, on the same assumptions as above, will converge to 0 ? And, if so, what is the maximum number by which we can replace the number " 2 " in this definition, for which we have convergence to 0 ?

It is hoped that these formal propositions can help better to understand actual choice situations; they have helped the authors better to understand the nature of conflicts and to shape their ideas as to the conditions under which conflicts do or do not arise. 
Notes

1 For a set $\mathrm{E}$, we denote by $|\mathrm{E}|$ its cardinal.

2 By $\subset$ we mean strict inclusion. The name "property B" is due to E. Miller ("On a Property of Families of Sets," Comptes Rendus, Varsavie 30, pp. 31-38, 1937), who used the letter B in honour of Felix Bernstein.

- By a maximal collection $\Gamma$ of subsets of an $n$-set satisfying (i), (iii), and (iv), we mean a collection with $|\Gamma|=2^{n-1}$.

- An extremal result is a result that sets a bound to $\mathrm{m}$, the number of different subsets.

- For a real number $x,[x]$ is the largest integer not exceeding $x$.

- For a set $\mathrm{S} \subset \mathrm{B}, \overline{\mathrm{S}}:=\mathrm{B} \backslash \mathrm{S}$.

- Let $f$ and $g$ depend on a parameter $x$, which tends, say, to a (in this paper $a=\infty$ ). Assuming that $g$ is positive, we write

$\mathrm{f}=0(\mathrm{~g})$, if $\mathrm{f} / \mathrm{g}$ remains bounded;

$f=o(g)$, if $f / g \rightarrow 0$;

$f \sim g$, if $\mathrm{f} / \mathrm{g} \rightarrow 1$.

- Erdös and Hindman ("Enumeration of intersecting Families," Discrete Mathematics 48 , pp $61-65,1984$ ) sharpened both the lower and upper bound to $g(n)$ presented here. Actually they proved $n$ $\log _{2}(g(n)) \sim\left(\left[\frac{\pi}{2}\right]\right) / 2$.

\section{References}

Arrow, K. J. (1963). Social Choice and Individual Values. New York: John Wiley.

Berge, C. (1970). Graphes et hypergraphes. Paris: Dunod.

Erdös, P. (1969). "On a Combinatorial Problem III." Canadian Mathematical Bulletin 12: 413-16.

Erdös, P., Chao Ko and R. Rado. (1961). "Intersection Theorems for Systems of Finite Sets." Quarterly Journal of Mathematics Oxford (2). 12: 313-20.

Erdös, P., and D. Kleitman. (1974). "Extremal Problems among Subsets of a Set." Discrete Mathematics 8: 281-94.

Erdös, P., and L. Lovász. (1975). "Problems and Results on 3-Chromatic Hypergraphs and Some Related Questions." In Infinite and Finite Sets, Vol., II. Ed. A. Hajnal, R. Rado, and V. T. Sos. Amsterdam: North-Holland. Pp. 609-27. I

Hilton, A. J. W. (1975). "Analogues of a Theorem of Erdös, Ko and Rado on a Family of Finite Sets." Quarterly Journal of Mathematics (Oxford) (2) 25: 19-28.

Katona, G. O. H. (1974). "Extremal Problems for Hypergraphs." In Combinatorics; Ed. M. Hall and J.H. van Lint.Mathematical Centre Tracts 56: $13-42$.

Kleitman, D. J., and G. Markowsky. (1975). "On Dedekind's Problem: The Number of Isotone Boolean Functions, II" Transactions of the American Mathematical Society 213: 373-90.

Koster, M. B. M. de, J. H. P. Paelinck, and P. H. Vossen. (1983). "Analysis and Measurement of Conflicts." To appear in: Proceedings of the World University Congress. Tokyo.

Milner, E. C. (1968). "A Combinatorial Theorem on Systems of Sets." Journal of the London Mathematical Society 43: 204-06.

Monjardet, B. (1978). "An Axiomatic Theory of Tournament Aggregation." Mathematics of Operations Research 3 (4): 334-51.

Moulin, H. (1983). The Strategy of Social Choice. Amsterdam: North-Holland. Sperner, E. (1928). "Ein Satz über Untermengen einer endlichen Menge." Mathematische Zeitschrift 27: 544-48. 